Commentary

\title{
Hepatic haemangioendothelioma: A proteiform disease
}

\author{
Elia Gigante $^{\mathrm{a}, \mathrm{b}}$, Quirino Lai ${ }^{\mathrm{c}}$, Jan P. Lerut ${ }^{\mathrm{d}}$, Jean-Charles Nault ${ }^{\mathrm{a}, \mathrm{e}, \mathrm{f}, *}$ \\ a Service d'hépatologie, Hôpital Jean Verdier, Hôpitaux Universitaires Paris-Seine-Saint-Denis, Assistance-Publique Hôpitaux de Paris, Bondy, France \\ ${ }^{\mathrm{b}}$ INSERM UMR 1149, Centre de recherche sur l'inflammation, Paris, France \\ ${ }^{c}$ Liver Transplant Program, Sapienza University of Rome, Rome, Italy \\ dinstitute for Experimental and Clinical Research [IREC], Université catholique Louvain (UCL), Brussels, Belgium \\ e Unité de Formation et de Recherche Santé Médecine et Biologie Humaine, Université Paris 13, Paris, France \\ ${ }^{\mathrm{f}}$ Centre de Recherche des Cordeliers, INSERM UMR 1148, Functional Genomics of Solid Tumors laboratory, Inserm, Sorbonne Université, Université Paris, \\ F-75006 Paris, France
}

\section{A R T I C L E I N F O}

Available online $\mathrm{xxx}$

Hepatic haemangioendothelioma (HEHE) is a rare vascular tumor developed in a normal liver. HEHE is a combination of epithelioid and histiocytoid vascular endothelial cells surrounded by fibrotic stroma with a pathognomonic CAMTA1/WWTR1 fusion at the molecular level [1]. Most patients present with a multinodular and bilobar liver disease (87\%); moreover, extrahepatic spread at the time of diagnosis is frequent (36.6\%) [2,3]. Most data about HEHE came from old series [4] or large series of patients treated by liver transplantation (LT) [5].

Unfortunately, reliable data on natural history and outcome of the different available treatments of HEHE are lacking.

In this issue of Digestive and Liver Diseases, SanduzziZamparelli et al. described a tri-centric retrospective cohort of 24 HEHE collected during the period 1994-2016; there was a female predominance $(66.7 \%)$, the median age was 38.7 years with various treatments applied (resection, transplantation, systemic treatment). Reported 5- and 10-year overall survival (OS) rates were $91.5 \%$ and $51.9 \%$. Eleven patients treated with LT or liver resection (LR) had 5- and 10 -year OS rates of $100 \%$ and $80 \%$ vs. $100 \%$ and $66.7 \%$, respectively. Five- and 10-year OS rates in six patients treated with chemotherapy were $83.3 \%$ and $62.5 \%$. In the case of abstention of any treatment $(n=7), 5$ - and 10 -year OS rates were $83.3 \%$ and $41.7 \%[6]$.

First, these results are in line with previously reported experiences, confirming that LT, when possible, represents the preferred

\footnotetext{
* Corresponding author at: Service d'hépatologie, Hôpital Jean Verdier, Hôpitaux Universitaires Paris-Seine-Saint-Denis, Assistance-Publique Hôpitaux de Paris Bondy, France

E-mail address: jean-charles.nault@aphp.fr (J.-C. Nault).
}

treatment, consenting to obtain excellent long-term survival rates in patients usually with more advanced disease [5].

Among 13 histological samples, 10 HEHE expressed Ki67<10\%, underlying the low proliferative index of most of HEHE. The authors found no significant difference in survival, whatever tumor number, size, and histological features. However, some caution must be taken when interpreting this information, as the Ki67 value is available in $46 \%$ of the studied patient cohort.

One of the samples was reclassified as hepatic angiosarcoma (HAS) after pathological review. This aspect highlights the challenge of differentiating HEHE from HAS. FISH or RT-PCR to detect the CAMTA1/WWTR1 fusion, identified only in this cancer, are potentially useful to confirm the diagnosis of HEHE [1,7]. In addition to histological and immunohistochemical assessment, genetic characterization combined with clinical features and the selection by time allows making a correct diagnosis. A mandatory waiting time of four months in patients waiting for LT is suggested with the intent to avoid misdiagnosis with HAS [5]. It is critical to make the right diagnosis, as HAS represents an absolute contraindication of LT.

Several data reported in the presented cohort differ from the literature. First, chronic liver diseases were present in $20 \%$ of patients (HCV- and alcoholic-related cirrhosis). Secondly, the number of asymptomatic patients at diagnosis was very high (68\% vs. $25 \%)$ [2]. This difference is potentially explained by a more frequent incidental diagnosis using modern imaging techniques [2].

Thirdly, the treatment modalities differ from previous reports; few patients had LT and more LR. A pooled analysis of HEHE publications shows that the most frequently used approach to HEHE is LT (44.8\%), followed by treatment abstention (24.8\%), chemo- or radiotherapy (21\%), and LR (9.4\%). One- and 5-year OS in patients 
not receiving any treatment were $39.3 \%$ and $4.5 \%$, respectively [2]. It must also be underlined that, due to the natural evolution of HEHE, LR is only possible in a minority (less than 10\%) of patients [2].

Finally, no patient presented at imaging signs of macrovascular invasion (MaVI), although $20 \%$ of patients had this condition at the pathological examination of the resected liver specimen. In previous experiences, the reverse was observed: higher MaVI percentages (40\%) at imaging but only $13 \%$ at pathological examination [5]. The low concordance between radiology and pathology findings suggests that vascular infiltration at imaging should be interpreted with caution, as clear radiological patterns of MaVI lack in this pathology.

Considering the retrospective nature and the small number of patients typically observed in the studies on HEHE, several questions concerning the best therapeutic option to choose should be raised. Sanduzzi-Zamparelli et al. confirm the well-known feature that a subset of HEHE present non-aggressive tumors explaining long-term survival rates without any treatment. Moreover, the authors confirmed that non-resectable/non-transplantable patients might benefit from systemic (although not yet standardized) chemotherapy to reach very variable 5-year survival rates (range from $3 \%$ to $43 \%$ ) $[2,8]$.

One study suggested that chemotherapy in non-surgical patients decreased 5-year survival rate (43.6\%) compared to patients without treatment (82.9\%) [8]. These differences in survival can be explained by the very different levels of HEHE aggressiveness, but also by selection biases caused by the small numerosity of the investigated studies.

In contrast to hepatocellular and cholangiocellular carcinoma, confined extrahepatic metastasis is not a useful marker to identify the aggressiveness of HEHE. It has been shown that LT with or without complementary surgery may consent to obtain very satisfactory results [5]. The fact that one (single) patient of the studied cohort had a good outcome following LR despite lung metastasis confirm these observations.

Due to the protean clinical manifestation of HEHE, it is crucial to develop in the future better markers able to identify the different levels of aggressiveness. Very aggressive forms (unfortunately often corresponding to a misdiagnosed HAS) are probably bad candidates for LR and LT. Slowly progressing HEHE benefit from LT and /or LR. In some cases, careful follow-up can confirm disease stability over a long-time period.

In the next future, it is highly desirable to identify different sub-types of HEHE using molecular biology and immunohistochemistry, in order to avoid tumor overtreatment (LT) and, more importantly, undertreatment (long follow-up without any treatment denying access to a possible curative surgical therapy).

It is important to collect more data in larger HEHE patient cohorts with the intent to investigate detailed pathological and molecular biology aspects, in order to "dissect out" the bio-clinical heterogeneity in HEHE patients and to refine the therapeutic algorithm of this rare disease.

\section{Declaration of Competing Interest}

Jean Charles Nault received a research grant from Bayer for Inserm UMR1148, Elia Gigante nothing to declare, Quirino Lai nothing to declare, Jan Lerut nothing to declare.

\section{References}

[1] Studer LL, Selby DM. Hepatic Epithelioid Hemangioendothelioma. Arch Pathol Lab Med 2018;142:263-7. doi:10.5858/arpa.2016-0171-RS.

[2] Mehrabi A, Kashfi A, Fonouni H, Schemmer P, Schmied BM, Hallscheidt P, et al. Primary malignant hepatic epithelioid hemangioendothelioma. Cancer 2006;107:2108-21. doi:10.1002/cncr.22225.

[3] Ganeshan D, Pickhardt PJ, Morani AC, Javadi S, Lubner MG, Elmohr MM, et al. Hepatic hemangioendothelioma: CT, MR, and FDG-PET-CT in 67 patients-a biinstitutional comprehensive cancer center review. Eur Radiol 2020;30:2435-42. doi:10.1007/s00330-019-06637-3.

[4] Ishak KG, Sesterhenn IA, Goodman ZD, Rabin L, Stromeyer FW. Epithelioid hemangioendothelioma of the liver: a clinicopathologic and follow-up study of 32 cases. Hum Pathol 1984;15:839-52. doi:10.1016/s0046-8177(84)80145-8.

[5] Lai Q, Feys E, Karam V, Adam R, Klempnauer J, Oliverius M, et al. Hepatic epithelioid hemangioendothelioma and adult liver transplantation: proposal for a prognostic score based on the analysis of the ELTR-ELITA registry. Transplantation 2017;101:555-64. doi:10.1097/TP.0000000000001603.

[6] Sanduzzi-Zamparelli M., Rimola J., Montironi C., Nunes V., Avancini Ferreira Alves V., Sapena V., et al. Hepatic epithelioid hemangioendothelioma: an international multicenter study. Dig Liver Dis Accept- Press n.d.

[7] Tanas MR, Sboner A, Oliveira AM, Erickson-Johnson MR, Hespelt J, Hanwright PJ, et al. Identification of a disease-defining gene fusion in epithelioid hemangioendothelioma. Sci Transl Med 2011;3 98ra82. doi:10.1126/scitranslmed.3002409.

[8] Thomas RM, Aloia TA, Truty MJ, Tseng WH, Choi EA, Curley SA, et al. Treatment sequencing strategy for hepatic epithelioid haemangioendothelioma. HPB 2014;16:677-85. doi:10.1111/hpb.12202. 